\title{
An Integrated Surgical Management for Giant Dermatofibrosarcoma Protuberans of Anterior Abdominal Wall
}

\author{
Swagata Brahmachari ${ }^{1}$, Anubha Pandey ${ }^{2}$, Mahendra Pratap Singh ${ }^{1}$, Vandana Agarwal ${ }^{3}$ \\ 1. Department of General Surgery, All India Institute of Medical Sciences, Bhopal, IND 2. Department of Pathology, \\ Atal Bihari Vajpayee Government Medical College, Vidisha, IND 3. Department of Pathology, LN Medical College and \\ Research Centre, Bhopal, IND
}

Corresponding author: Swagata Brahmachari, swagata.surgery@aiimsbhopal.edu.in

\begin{abstract}
Giant dermatofibrosarcoma protuberans (DFSP) is a very rare dermal sarcoma whose diagnosis and management are important because of the high local recurrence but low metastatic potential. Complete surgical excision of giant DFSP in a single stage is difficult but has a high cure rate.

A 47-year-old man presented with a gradually increasing large $(18 \times 15 \times 7 \mathrm{~cm})$ DFSP in the epigastrium. A 3 $\mathrm{cm}$ circumferential wide local excision (WLE) with microscopic tumor-free margin confirmed by frozen section was performed. Immediate single staged tension-free primary closure of resultant defect was done on the principle of abdominal wall reconstruction (AWR) in ventral hernia repair. This technique of anterior component separation and bridge meshplasty is functional, avoids multiple surgeries, is cost-effective, and can be done in a resource-limited setting in developing countries.
\end{abstract}

A multidisciplinary and integrated surgical approach to treat giant DFSP over epigastrium, by threedimensional WLE and immediate AWR with anterior component separation technique (CST) and bridging meshplasty, can be of immense help in managing such rare cases in developing countries.

Categories: Plastic Surgery, General Surgery, Oncology

Keywords: synthetic mesh, dermatofibrosarcoma protuberans, giant, three-dimensional excision, abdominal wall reconstruction, component separation technique

\section{Introduction}

Dermatofibrosarcoma protuberans (DFSP) are rare, cutaneous mesenchymal neoplasm with an annual global incidence of 0.8-4.5 cases per million persons. Hoffman named this rare clinical entity DFSP which accounts for $1-6 \%$ of soft tissue sarcomas and $18 \%$ of cutaneous soft tissue sarcomas [1]. DFSP is a superficial, slowgrowing, locally aggressive, infiltrative cutaneous sarcoma arising from the dermis. It is composed of CD-34 positive, neoplastic spindle cells with high local recurrence and low metastatic potential [2]. Large-sized DFSP and delayed presentation are often due to rarity and misdiagnosis as a benign tumor because of its indolent evolution. Usually, it presents as a 1-6 cm-sized tumor, but rarely $>20 \mathrm{~cm}$ have also been reported [2]. Clinical awareness of this uncommon oncological entity is important as complete surgical excision with histologically negative margin has an excellent prognosis with a $99 \%$ five-year survival rate [3]. Repair of the resultant defect in the anterior abdominal wall is necessary to maintain function. So we present an integrated surgical approach to treat a giant DFSP over epigastrium by three-dimensional wide local excision (WLE) and immediate abdominal wall reconstruction (AWR) by anterior component separation technique (CST) with bridging meshplasty.

\section{Case Presentation}

A 47-year-old man presented with a slow-growing large tumor in the epigastrium for four years with occasional pain. Clinically, a 15 x 14 cm large, lobulated, firm, non-tender tumor covered by shiny telangiectatic skin was present over the anterior abdominal wall of the epigastrium (Figure $1 \mathrm{~A}$ ). Radio imaging studies (contrast-enhanced computed tomography [CECT], MRI) revealed a large, heterogeneous, exophytic tumor measuring $16.4 \times 14.6 \times 9.7 \mathrm{~cm}$ over the anterior abdominal wall of epigastrium, adherent to skin, subcutaneous tissue, and underneath rectus muscle in the midline, without any visceral metastasis or regional lymphadenopathy (Figure $1 B$ ). Complete surgical excision with immediate reconstruction was planned by an integrated multidisciplinary team, as the patient was unable to afford neoadjuvant imatinib therapy. A three-dimensional excision of the tumor of size $18 \times 15 \times 10 \mathrm{~cm}$, with a three-centimeter macroscopic tumor-free margin circumferentially, including underlying rectus sheath and peritoneum, was performed (Figure 1C). Frozen section evaluation showed negative microscopic peripheral and deep margin. Further, one cm shaved peripheral margin along with the en-blocked excised tumor was sent for histopathological evaluation. The resultant full-thickness abdominal wall defect of size $22 \times 19 \mathrm{~cm}$ (Figure 1D) was reconstructed by modified anterior CST with bridging meshplasty. 


\section{Cureus}
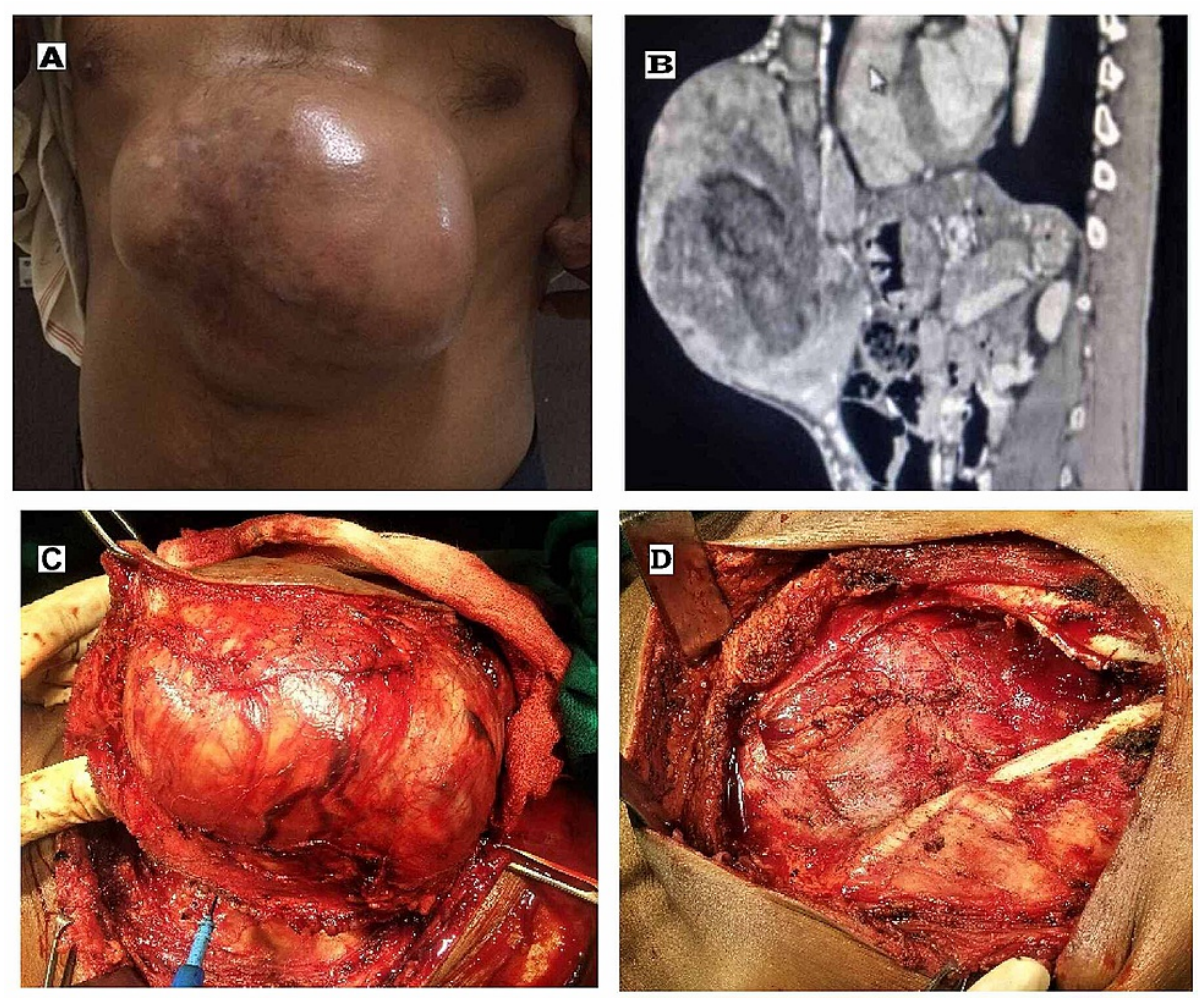

\section{FIGURE 1: Gross and perioperative view of dermatofibrosarcoma}

protuberans.

1(A): A large protuberant mass located in the epigastrium.

1(B): MRI (chest and abdomen) showing large heterogenous mass $(16.4 \times 14.6 \times 9.7 \mathrm{~cm})$ on the anterior abdominal wall in the epigastric region, arising from rectus abdominis muscle, and the gastric antrum appears adherent to the deeper margin of the lesion.

1(C): Three-dimensional wide local excision being done with $3 \mathrm{~cm}$ macroscopic clear margin.

1(D): Resultant full-thickness abdominal wall defect $(24 \times 21 \mathrm{~cm})$ following en-block excision of tumor in the epigastrium and the peritoneal defect repaired.

After primary peritoneal closure, a preperitoneal polypropylene mesh was sutured to the defect margin of the posterior rectus sheath as a bridging mesh for a tension-free repair (Figure $2 \mathrm{~A}$ ). Then, by anterior CST, a myofascial flap of anterior abdominal wall muscles from both sides was approximated medially over the mesh along the oblique incision line to fill the defect, and the abdomen closed in a single layer with suction drainage (Figure 2B). Microscopically, monotonous, spindle-shaped tumor cells arranged in storiform patterns with well-differentiated fat cells entrapped within the spindle cells were seen in the subcutaneous layer with a superficial Grenz zone separating the normal epidermis (Figure 2C). All the margins were free of tumor cells. Ki67 was focally positive in $2-4 \%$ of tumor cells, but areas of sarcomatous transformation were not found. Immunohistochemistry (IHC) showed strongly positive CD34 and vimentin and negative for smooth muscle actin (SMA) and S-100, confirming the diagnosis of DFSP (Figure 2D). Post-operative recovery was uneventful. No local recurrence or metastases were found during regular follow-up for the last five years. 


\section{Cureus}
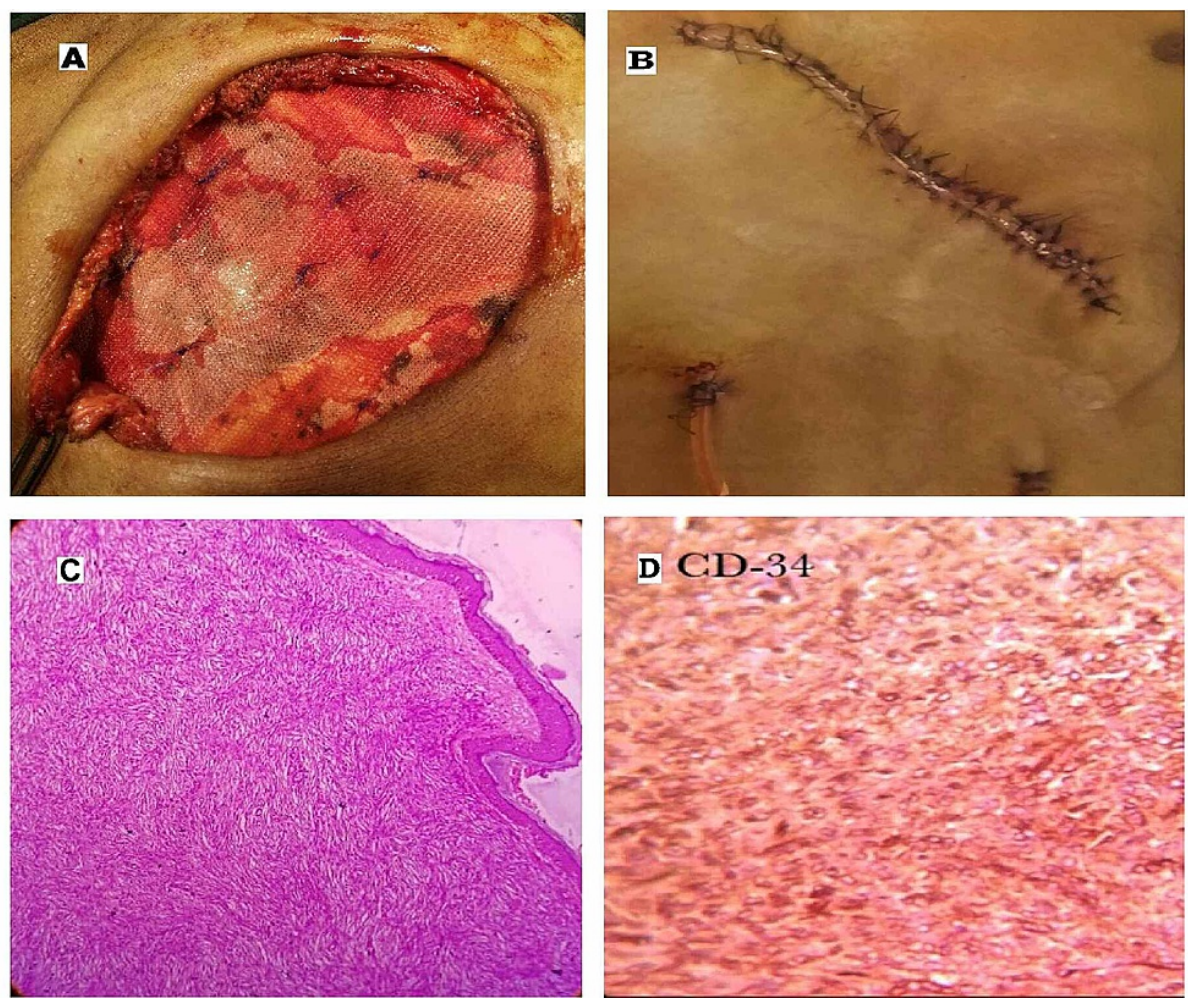

\section{FIGURE 2: Defect in abdominal wall post excision confirmatory microscopic view.}

2(A): Reconstruction of the anterior abdominal wall done by suturing polypropylene mesh to defect margin preperitoneally.

2(B): Full-thickness abdominal wall reconstruction with skin flap approximation done by anterior component separation over the bridging mesh for functional and aesthetic repair.

2(C): The histopathological section showing spindle-shaped tumor cells in the dermis arranged in a storiform pattern. Free Grenz zone with the normal epidermis, along with infiltration with tumor cells into the subcutaneous tissue (HE staining 40X).

2(D): Immunohistochemistry of tumor cells showing cluster of differentiation for CD 34 antigen (magnification 40X).

\section{Discussion}

DFSP is a superficial fibroblastic dermal sarcoma of intermediate malignancy. The pathogenesis is still uncertain but $10-20 \%$ have a history of trauma and in $80-90 \%$ of cases, there is somatic mutation by a protooncogene COL1A1-PDGFB. This is formed by translocation of 17 and 22 chromosomes, which causes activation of the tyrosine-kinase pathway leading to PDGF $\beta$ overexpression [4].

DFSP mostly presents as a slow-growing, painless, superficial, lobulated, and protuberant tumor over the trunk (40-50\%) among the 20-50 years age group. The overlying skin is thin, shiny, telangiectatic, and adherent to the tumor [2]. Radio imaging (CT and MRI) is useful for preoperative evaluation, surgical planning, and diagnosis of distant metastasis and recurrence $[3,5]$.

Distinctive histopathological findings of DFSP are spindle-shaped neoplastic cells arranged in a storiform or cartwheel pattern in the dermis, with neoplastic tentacles extending into the surrounding subcutaneous tissues [5]. Hence, tumor resection with a negative margin is difficult, resulting in high local recurrence. Immunohistochemical staining, showing strong positivity of CD34 and vimentin and negative S-100, is diagnostic of DFSP [6,7]. Sarcomatous changes occurring in 10-15\% of DFSP, are associated with a giant size and poor prognosis in comparison to classical DFSP [6]. Our case is a giant classical DFSP but without fibrosarcomatous changes, which is very rare.

Very few studies are reported on the management of abdominal wall DFSP. Complete surgical excision with histologically negative margins is the standard treatment of DFSP [5]. This can be achieved by WLE or Mohs micrographic surgery depending on the size, location of the tumor, and availability of expertise [5]. As per 
National Comprehensive Cancer Network (NCCN) guidelines (version 1.2020), the standard recommendations for improving recurrence-free survival is complete three-dimensional WLE of the tumor with 2-4 cm negative histological margin along with skin, surrounding tissue, and underlying deep fascia $[3,5]$. In truncal DFSP and resource-challenged centers, WLE is preferred because of easy expertise availability, less time-consuming, cost-effective, and in a single stage, excision with reconstruction can be done with excellent cosmetic and functional outcomes $[2,5,8]$. The absence of neoplastic cells on margins is the most important prognostic factor for local recurrence [7]. In this case, microscopic negative margins were ensured by three-dimensional excision with three centimeters wide surgical margin, peri-operative frozen section margin evaluation, and an additional shaved margin for histopathological evaluation. This protocol of excision of an additional $1 \mathrm{~cm}$ to the standard $3 \mathrm{~cm}$ margin in WLE of huge $(>5 \mathrm{~cm})$ DFSP along with immediate reconstruction after negative frozen section resection margins, has been done by Kim BJ in Korea with very few recurrences [7].

After WLE, the reconstruction of the resultant full-thickness defect aims to protect the abdominal viscera, restoring the functional integrity of the abdominal wall along with its aesthetic appearance and hernia prevention $[9,10]$. Reconstruction of Type II and III large abdominal wall defects (> $6 \mathrm{~cm}$ in diameter) is generally done by vascularized autologous free or pedicled flaps that need the expertise of plastic surgeons [9].

AWR by CST is a recently developed technique of ventral hernioplasty with the principle of re-establishing a functional abdominal wall with autologous tissue repair. This technique can be modified and used in the reconstruction of post-oncologic full-thickness abdominal wall defects after abdominal DFSP excision $[9,10]$. So primary repair of full-thickness abdominal wall defect of Type III C, after WLE for DFSP, can be achieved by CST as it minimizes the defect size by the advancement of musculofascial layer over the bridging mesh used to cover the defect $[9,11]$. Advances in minimal invasive CST techniques, such as perforator sparing anterior CST, endoscopic CST, minimally invasive posterior CST, help in decreasing the postoperative wound complications [11]. But this technique is of limited use in the closure of large defects of Type C, of size more than $40 \mathrm{~cm} 2$ with wide loss of domain, wherein free musculo fasciocutaneous flaps or vascularized pedicled flaps are preferred [9].

In the reported case, the resultant abdominal wall defect was repaired by AWR with anterior CST and bridge meshplasty for a single staged, tension-free primary functional reconstruction, customized as per the position of the defect. This technique is functional, cost-effective, avoids multiple surgeries, and can be done in a resource-limited setting even by experienced general surgeons. The aim was to simplify the surgical approach, decrease the operative time, cost, morbidity of multiple surgeries, and thus infection rate. So by the preoperative marking of tumor extent with MRI, WLE with $3 \mathrm{~cm}$ clear lateral and deep margins, preoperative frozen section histology along with extra shaved margins, aids in achieving oncological negative margins resection. A functional reconstruction can be achieved by AWR by anterior CST and meshplasty.

In recurrent lesions or positive margins, re-excision followed by adjuvant radiotherapy or targeted therapy with tyrosine kinase inhibitors like imatinib is advocated [7]. The prognosis of DFSP after adequate surgical excisions is very good. A longer duration of follow-up, over five years, has been associated with decreased morbidity and mortality [2]. Recurrences are seen mostly within three years but may occur later, so longterm follow-up is mandatory at an interval of 6-12 months [5].

\section{Conclusions}

Awareness of DFSP, a rare surgically curable but locally aggressive dermal sarcoma, is important for a proper diagnosis and management to prevent recurrences. An integrated approach, involving surgery, histopathology, and immuno-radiotherapy, can act as an elixir for making DFSP a curative entity. Anterior CST with meshplasty is an accepted hernia repair technique that can be used for immediate AWR after oncological resection of DFSP with good results by general surgeons in resource-limited places. So to improve the functional and aesthetic outcome without compromising on oncological safety and hernia prevention, a modified three-dimensional excision of the tumor with deep fascia and $3 \mathrm{~cm}$ circumferential safe surgical margin, confirmed by frozen section with immediate reconstruction by CST with mesh reinforcement is recommended by us in full-thickness abdominal wall defects following DFSP resection.

\section{Additional Information}

\section{Disclosures}

Human subjects: Consent was obtained or waived by all participants in this study. Conflicts of interest: In compliance with the ICMJE uniform disclosure form, all authors declare the following: Payment/services info: All authors have declared that no financial support was received from any organization for the submitted work. Financial relationships: All authors have declared that they have no financial relationships at present or within the previous three years with any organizations that might have an interest in the submitted work. Other relationships: All authors have declared that there are no other relationships or activities that could appear to have influenced the submitted work. 


\section{Cureus}

\section{References}

1. Brooks J, Ramsey ML: Dermatofibrosarcoma Protuberans. StatPearls Publishing, Treasure Island, Florida; 2021.

2. Malan M, Xuejingzi W, Quan SJ: The efficacy of Mohs micrographic surgery over the traditional wide local excision surgery in the cure of dermatofibrosarcoma protuberans. Pan Afr Med J. 2019, 33:297. 10.11604/pamj.2019.33.297.17692

3. Wiesmueller F, Agaimy A, Perrakis A, Arkudas A, Horch RE, Grützmann R, Vassos N: Dermatofibrosarcoma protuberans: surgical management of a challenging mesenchymal tumor. World J Surg Oncol. 2019, 17:90. 10.1186/s12957-019-1627-3

4. Dhir M, Crockett DG, Stevens TM, Silberstein PT, Hunter WJ, Foster JM: Neoadjuvant treatment of Dermatofibrosarcoma Protuberans of pancreas with Imatinib: case report and systematic review of literature. Clin Sarcoma Res. 2014, 4:8. 10.1186/2045-3329-4-8

5. Hao X, Billings SD, Wu F, Stultz TW, Procop GW, Mirkin G, Vidimos AT: Dermatofibrosarcoma protuberans: update on the diagnosis and treatment. J Clin Med. 2020, 9:1752. 10.3390/jcm9061752

6. Larbcharoensub N, Kayankarnnavee J, Sanpaphant S, Kiranantawat K, Wirojtananugoon C, Sirikulchayanonta V: Clinicopathological features of dermatofibrosarcoma protuberans . Oncol Lett. 2016, 11:661-667. 10.3892/ol.2015.3966

7. Kim BJ, Kim H, Jin US, Minn KW, Chang H: Wide local excision for dermatofibrosarcoma protuberans: a single-center series of 90 patients. Biomed Res Int. 2015, 2015:642549. 10.1155/2015/642549

8. Song Z, Yang D, Yang I, Nie X, Wu J, Song H, Gu Y: Abdominal wall reconstruction following resection of large abdominal aggressive neoplasms using tensor fascia lata flap with or without mesh reinforcement. Hernia. 2018, 22:333-341. 10.1007/s10029-018-1738-8

9. Spera LJ, Danforth RM, Hadad I: Incisions and reconstruction approaches for large sarcomas . Transl Gastroenterol Hepatol. 2018, 3:86. 10.21037/tgh.2018.10.07

10. Scheuerlein H, Thiessen A, Schug-Pass C, Köckerling F: What do we know about component separation techniques for abdominal wall hernia repair?. Front Surg. 2018, 5:24. 10.3389/fsurg.2018.00024

11. Mericli AF, Baumann DP, Butler CE: Reconstruction of the abdominal wall after oncologic resection: defect classification and management strategies. Plast Reconstr Surg. 2018, 142:187S-196S.

10.1097/PRS.0000000000004877 